\title{
Tromboembolismo pulmonar en pacientes médicos. Aproximación a los costes hospitalarios y tendencias evolutivas en España
}

\author{
J. MONTES SANTIAGO'1, G. REY GARCÍA², A. MEDIERO DOMÍNGUEZ² \\ ${ }^{I}$ Servicio de Medicina Interna. ${ }^{2}$ Servicio de Documentación Clínica. Hospital Meixoeiro. \\ Vigo. Pontevedra
}

PULMONARY EMBOLISM IN MEDICAL INPATIENTS. AN APPRO-
ACH TO TRENDS AND COSTS IN SPAIN

\section{RESUMEN}

Fundamento: La generalización en los últimos años de tromboprofilaxis en pacientes hospitalizados haría esperable una disminución de casos de tromboembolismo pulmonar (TEP). Evaluamos la evolución del TEP en el Servicio Nacional de Salud (SNS) durante el periodo 19971999, estimándose sus costes para el año 1999.

Método: A partir del Conjunto Mínimo Básico de Datos (CMBD) de hospitales del SNS estudiamos el Grupos Relacionados de Diagnóstico (GRD) 78 (Embolismo pulmonar), anotándose edad, sexo y evolución anual. Tales datos se refieren fundamentalmente a pacientes médicos. El coste se calculó multiplicando número de episodios por coste medio de este GRD establecido por el Ministerio de Sanidad y Consumo para 1999. Ya que el incremento de tendencia podría sólo reflejar mejoría en tasas de declaración o mayor incorporación de hospitales declarantes al CMBD, comparamos los datos con los procedentes del Servicio Gallego de Salud (SERGAS), cuya tasa de codificación de altas supera el $96 \%$.

Resultados: Fueron observados 14.021 casos en el SNS en dicho periodo. El $47 \%$ ocurrió en pacientes $>75$ años. En 1999 la mortalidad fue del $6,8 \%$. Tanto en SERGAS como en SNS aumentaron las hospitalizaciones anuales por TEP (5\% en 1999 respecto a 1997). Para 1999 el coste se estimó en 16-20,2 millones $\quad(0,097 \%-0,12 \%$ del gasto sanitario en atención especializada).

Conclusiones: Se observa una tendencia anual creciente de casos de TEP en España. Si esto refleja mayor número de declaraciones al CMBD o aumento real de incidencia está por dilucidar aunque los datos del SERGAS sugieren la segunda posibilidad.

\begin{abstract}
Background: Tromboprophylaxis for hopitalized patients has became a widespread practice in the lasts years. However, the trends in pulmonary embolism (EP) are insufficiently known. We analyzed the trends of EP during the 1997-1999 period in Spain at the hospitals of the National Health System (NHS) and theirs costs were also estimated.

Methods: Diagnosis-Related Group (DGR) 78 from the data of the national hospital discharge register was evaluated. Annual trends, age, sex and (for 1999) mortality, some comorbidities and costs according official data from the Spanish Ministry of Health were annotated. Mostly medical patients were included. Data were compared with those from the Servicio Gallego de Saúde (SERGAS) which maintain a high rate of hospitalary discharges declaration.

Results: During the 3-year period 14.021 cases of EP were observed. The 47\% of the NHS cases were older than 75 and mortality was 6,8\%. An annual increment of hospitalized cases was observed at SERGAS and NHS (in the last 5\% at the end of the period). In 1999 costs were estimated in 16-20,2 millions. That was 0,097-0,12\% of global hospitalary budget.

Conclusions: Trends for hospitalizations for EP are increasing in Spain. It remains in doubt if a true increase in incidence or the improving notification and awareness are the responsibles for this increment. However, the data of SERGAS support the first possibility.
\end{abstract}

KEY WORDS: Pulmonary embolism. Inpatients costs.

PALABRAS CLAVE: Tromboembolismo pulmonar. Costes hospitalarios.

Montes Santiago J, Rey García G, Mediero Domínguez. A. Tromboembolismo pulmonar en pacientes médicos. Aproximación a los costes hospitalarios y tendencias evolutivas en España. An Med Interna (Madrid) 2004; 21: 326-330.

\section{INTRODUCCIÓN}

El tromboembolismo pulmonar (TEP) constituye un proceso grave y frecuentemente infradiagnosticado, habiéndose encontrado en series autópsicas una prevalencia de hasta el
$14 \%$, en la mayor parte no sospechados en vida (1). Se ha comunicado que más del $90 \%$ de las muertes por TEP ocurren en aquellos pacientes no tratados porque su diagnóstico no llegó a realizarse (2). De hecho, en las series con mejores resultados el diagnóstico antemortem sólo se realiza en torno al

Trabajo aceptado: 23 de febrero de 2004 
$50 \%$ de casos $(1,3)$. Sin embargo, su reconocimiento y tratamiento precoz permiten disminuir la mortalidad desde el $30 \%$ a menos del $5 \%(2,4,5)$. Su incidencia es muy variable, comunicándose cifras entre el 0,6-1,8 casos por 1.000 personasaño, habiéndose observado una dependencia acusada de la edad, estimándose que por cada década su incidencia se dobla, hasta alcanzar cerca del $11 \%$ a los 80 años $(6,7)$. La incidencia entre pacientes hospitalizados es 100 veces mayor que la de los pacientes ambulatorios (8). Anualmente se calculan en Francia unos 100.000 casos, 60.000 en Italia, 65.000 en pacientes hospitalizados en el Reino Unido y 250.000 casos en EE.UU. $(9,10)$. En este último país, se estiman los costes derivados de la enfermedad tromboembólica venosa en unos 1.500 millones de dólares/año (11).

En nuestro país, como se ha apuntado recientemente (12), son escasos los estudios epidemiológicos que aborden este problema y las cifras aportadas en publicaciones recientes -65.000 casos de ETV y 25000 EP/año (13)- reflejan meramente extrapolaciones a nuestro medio de datos de otros países. Últimamente, citando datos procedentes del Sistema Nacional de Salud (SNS) en el bienio 1998-99 se ha apuntado que su incidencia podría estar en torno a los 7 casos por 10.000 habitantes/año (lo cual arrojaría unos 28.000 casos/año), si bien la cifra real podría doblarse debido a los casos asintomáticos (14). Su mortalidad se situó en el 7,5\%, similar a la mortalidad encontrada en otros países occidentales que oscila entre el 2 y $8 \%$ (15). En otro estudio que recoge 1.745 pacientes estudiados en un solo centro entre 1993 y 2001, se confirma que la incidencia aumenta con la edad hasta alcanzar un pico en la década de los 70 años (12). Además en este grupo de edad la mortalidad es mayor como se ha evidenciado en algunas series recientes de nuestro país $(16,17)$.

La introducción generalizada de prácticas de tromboprofilaxis en servicios quirúrgicos y en pacientes médicos con alto riesgo haría esperable una disminución de los casos de TEP, pero esta característica ha sido escasamente estudiada. La publicación del Conjunto Mínimo Básico de Datos (CMBD) del SNS, su agrupación en Grupos Relacionados de Diagnósticos (GRDs) y su facilidad de acceso a través de Internet, proporcionan una oportunidad única para examinar diversos aspectos de dicha entidad. Por ello, nuestro objetivo fue evaluar la evolución del TEP según los datos procedentes del Servicio Nacional de Salud (SNS) durante el periodo 1997-1999, y aproximar mediante ellos los costes de hospitalización de este proceso.

\section{MATERIAL Y MÉTODO}

A partir del CMBD de los hospitales del SNS (18) se estudió el GRD 78 (Embolismo pulmonar), anotándose la evolución anual y características de edad y sexo para el periodo 1997-1999. Para el año 1999 los datos utilizados fueron los Estadísticos de Referencia Estatal del SNS, los cuales además proporcionan información sobre los hospitales que declaran los casos (agrupados según su número de camas) y sobre procesos comórbidos asociados (19). El coste se calculó multiplicando el número de episodios por el coste medio del GRD 78 en 1999 (3.282 ) (19). Es importante resaltar que este estudio debe considerarse representativo fundamentalmente de los pacientes médicos con TEP. El CMBD puede infraestimar de forma apreciable los casos de TEP en pacientes ingresados en servicios quirúrgicos puesto que, en estos, las codificaciones de alta se refieren, obviamente, a GRDs quirúrgicos y sólo excepcionalmente a GRDs médicos como el 78. Una estimación aproximada de esta infranotificación de casos totales de TEP se situaría en 3.107 casos $(21 \%)$. Se puede asumir que dichos casos ocurrieron mayoritariamente en pacientes quirúrgicos. Dicha cifra se obtiene detrayendo del número de altas con los códigos de diagnósticos de la Clasificación Internacional de Enfermedades, $9^{a}$ Modificación clínica (CIE-9MC) 415.1 (embolismo pulmonar e infarto) y 415.19 (Otros embolismos pulmonares) (17.128 altas) el número de altas dentro del GRD 78 (14.021 altas), en el trienio considerado. No fueron considerados aquí los TEP en procesos obstétricos. Ya que el aumento de la tendencia evolutiva podría sólo reflejar mejoría en las tasas de declaración o mayor incorporación de hospitales declarantes al CMBD, se utilizaron como comparación datos procedentes de los hospitales públicos del Servicio Gallego de Salud (SERGAS) (20). Dicho organismo mantiene desde el año 1996 una tasa de codificación de altas válidas $>96 \%$ y, por tanto, el aumento de casos reflejaría un aumento real de hospitalizaciones más que aumento de las tasas de declaración.

\section{RESULTADOS}

En la figura 1 se muestran los resultados del SNS y en la figura 2 los resultados del SERGAS. El 47\% de los 14.021 casos del periodo 1997-1999 en el SNS ocurrió en pacientes $>75$ años. En este grupo etario el $60 \%$ fueron mujeres. Se

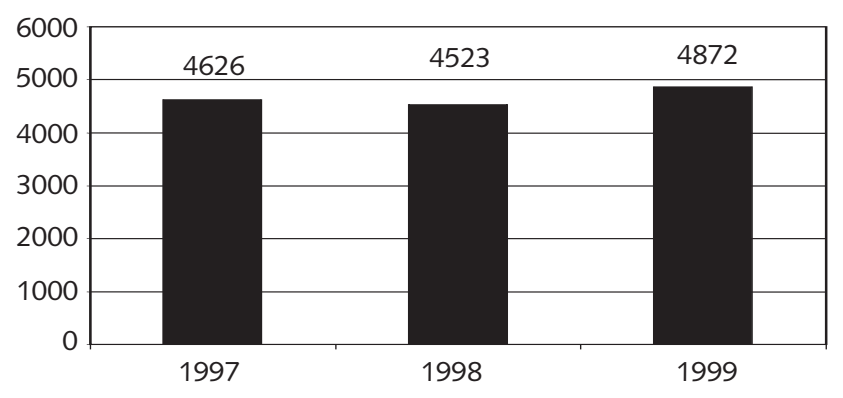

Fig. 1. Evolución de los casos de embolismo pulmonar (GDR 78) en el Sistema Nacional de Salud.

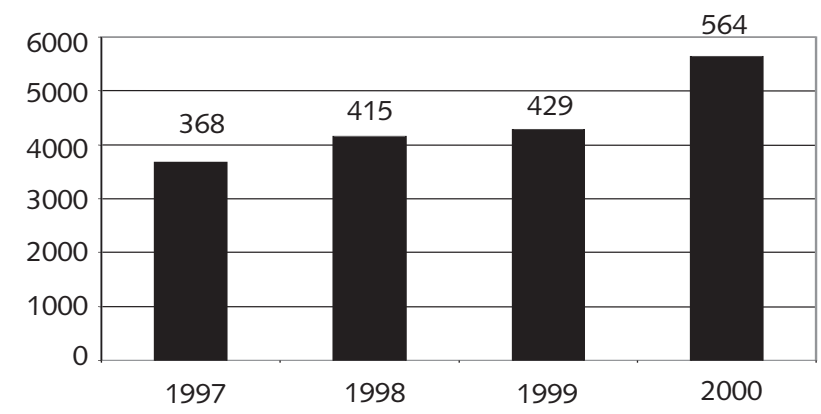

Fig. 2. Evolución de los casos de TEP de el Servicio Gallego de Salud. 
observa una tendencia creciente en el número de casos hospitalizados. Respecto al año 1997 en 1999 en el SNS se observó un incremento del 5,3\% en los casos de TEP. Para 1999 los casos de TEP (GDR 78) supusieron un 0,15/100 (IC95\%: 0,15-0,16) de altas válidas comunicadas al SNS (4.872/3.119.230) y el 0,20/100 (IC: 0,19-2,1) altas del SERGAS (429/216.093). En 1999 la mortalidad fue del 6,8\% y la estancia media de 12,9 días. En este año en el $17 \%$ se consignó la trombosis venosa en otros territorios y en el $12 \%$ la existencia de fibrilación auricular.

En la tabla I se realiza una estimación de los gastos por TEP (GDR 78) en el SNS. Para 1999 el coste de este proceso se estimó en 16 millones de , lo cual supuso el 0,097\% (16 /16555,6 millones ) (19) del gasto sanitario nacional en atención especializada. No obstante, si se admite que en cerca del $50 \%$ de casos ingresados de trombosis venosa profunda (TVP) puede asociarse un TEP silente $(11,22,23)$, entonces se obtendrían aproximadamente 7.038 casos de TEP en 1999, que constituyen 0,26/100 altas (IC: 0,25-0,26). Dicha cifra de TEP se obtiene al sumar los casos del GDR 78 (4.872) con el\% de casos en que el código CIE-9MC 453.8 (Trombosis de otras venas especificadas) contribuye a los GRDs 130 [Trastornos vasculares periféricos con complicaciones, $20 \%$ del total de este GRD, 1352 casos, coste medio: 2.428 ] y GRD 131 [Trastornos vasculares periféricos sin CC, $23 \%$ del total de este GRD, 2.981 casos, coste medio: 1.713 ], multiplicando por 0,5 los casos de ambos GRDs (676 casos en el GRD 130 y 1.490 en el GRD 131). Los 20,2 millones obtenidos supondrían el $0,12 \%$ de la asistencia especializada.

\section{DISCUSIÓN}

En años recientes se ha producido en los países occidentales un aumento generalizado de prácticas profilácticas destinadas a impedir el desarrollo de la enfermedad tromboembólica. Datos procedentes de prospecciones de mercado en varios de estos países (EE.UU., Francia, Alemania, Italia, España, Reino Unido y Japón) estimaron el gasto en agentes para prevenir o tratar la TVP/TEP en cerca de 1.300 millones $\$$ en 2001 y prevén para dichos fármacos una fuerte expansión en el próximo decenio. Dichas fuentes estimaron que en los países mencionados se produjeron cerca de 1,2 millones de casos de TVP/TEP y cerca de 52,7 millones eran individuos susceptibles de profilaxis (24). Estudios recientes han puesto además de relieve que dichas prácticas con heparinas de bajo peso molecular resultan coste-efectivas en cirugía ortopédica, tras traumatismos o en el tratamiento ambulatorio de las TVP (25). Encuestas sobre tromboprofilaxis procedentes de países como EE.UU., Canadá, Inglaterra, Suecia, Suiza, España y Australia/Nueva Zelanda, si bien documentan una amplia variedad de prácticas (entre el 28 y $100 \%$ de los respondedores realizan profilaxis sistemática), muestran, en general una sensibilización creciente por dicho problema (26). En un estudio reciente en nuestro país cerca del $58 \%$ de pacientes sometidos a cirugía general recibían tromboprofilaxis al alta (27).

Los datos anteriores contrastan, sin embargo, con estudios recientes en que se alerta sobre una creciente incidencia de diagnósticos de TEP en pacientes hospitalizados (28,29). En nuestro estudio hemos observado un aumento de la notificación de las hospitalizaciones por TEP tanto en el SERGAS como en el SNS. La cifra de 0,15 casos de TEP/100 hospitalizaciones en 1999 (SNS) o 0,20/100 (SERGAS) pueden considerarse cercanos a la incidencia reciente comunicada en EE.UU. (0,27\%; IC: 0,22-0,34\%) (28), en el periodo 1998-2000.

No obstante, es preciso considerar aquí las limitaciones de este estudio. Como ya se ha comentado el uso de este GDR médico puede conllevar una considerable infranotificación de los casos totales de TEP, estimada en cerca del $20 \%$, ya que dicho GDR apenas aparece reflejado en pacientes quirúrgicos. No obstante, y con esta advertencia en mente, hemos preferido realizar así este estudio debido a la notable complejidad que supone el cálculo de la parte del gasto atribuible al TEP en pacientes quirúrgicos con esta complicación. Hay que notar, además, que, aunque en el estudio estadounidense citado (28) se realizó una búsqueda más exhaustiva del diagnóstico de TEP mediante los Códigos CIE-9, los propios autores comentan la dificultad de estos para calcular la incidencia de TEP.

Dada la naturaleza administrativa de los datos de los que procede nuestro estudio es difícil decidir si esto supone un aumento real de la incidencia o un aumento de las declaraciones. El hecho de que esta tendencia se observe en el SERGAS que mantiene ya una tasa de codificación elevada de altas, y por tanto, con poco margen de mejora en sus tasas de declaración, sugiere la posibilidad de un aumento real de la incidencia. Esto puede deberse a una mayor sospecha diagnóstica en la detección de esta entidad, unido a la introducción en los últimos años de técnicas de imagen más sensibles en su diagnóstico, como el TAC helicoidal o RNM, que se han incorporado de forma creciente a los algoritmos diagnósticos $(9,14,30-35)$. Esto podría contribuir a explicar la paradoja del aumento de los diagnósticos a pesar del cada vez más extendido empleo de tromboprofilaxis en los servicios médicos y quirúrgicos. No obstante, es preciso notar que en el periodo considerado tales nuevas técnicas no estaban disponibles de modo generalizado en los hospitales del SNS, o al menos en aquellos hospitales < 500 camas y que contribuyeron al 44\% de los casos (2.129 de 4.872 en 1999).

TABLA I

ESTIMACIÓN DE LOS COSTES DE HOSPITALIZACIÓN POR TROMBOEMBOLISMO PULMONAR (TEP) EN 1999 EN ESPAÑA

\begin{tabular}{lcccc}
\hline & Denominación & Casos & Coste medio (€) & Total (millones $€$ ) \\
\hline GRD 78 & TEP & 4.872 & 3.282 & 15,99 \\
GRD 130 & TEP silente & 676 & 2.428 & 1,64 \\
GRD 131 & TEP silente & 1.490 & 1.713 & 2,55 \\
Total & & 7.038 & & 20,18 \\
\hline
\end{tabular}


Además de confirmar la avanzada edad de los pacientes hospitalizados con TEP (casi 1 de cada 2 son $>75$ años), la accesibilidad de las bases de datos nacionales permiten establecer otras comparaciones interesantes. Por ejemplo, según consta en el registro del SNS en el $17 \%$ de casos de TEP se señala la existencia de trombosis en otros territorios venosos (21). Aunque las bases no están diseñadas para cálculo de incidencias es interesante contrastar que la presencia de trombosis venosa concomitante en los casos de TEP que se busca específicamente puede oscilar entre el 13 y $93 \%$ de las series clínicas (35). Así mismo, nos permiten confirmar asociaciones de estudios procedentes de otras fuentes. Por ejemplo se han señalado como factores predisponentes en el TEP la obesidad, la hipertensión arterial o el tabaquismo $(36,37)$. En el CMBD del SNS el tabaquismo se consignó en el $17 \%$ de los casos de TEP y la hipertensión en el $25 \%$ (19). La puesta en marcha reciente de proyectos como el Registro RIETE con más de 80 centros nacionales participantes y con cerca de 8.000 pacientes introducidos proveerá abundante información que permita un adecuado manejo de estos pacientes (38).

En resumen, en este trabajo se observa una tendencia anual creciente del diagnóstico de TEP en pacientes médicos, aun a pesar de la generalización de prácticas tromboprofilácticas en estos. Si esto refleja un mayor número de declaraciones al CMBD o un aumento real de incidencia está por dilucidar aunque los datos del SERGAS sugieren la segunda posibilidad. Tal proceso constituye un gravoso problema económico estimado en cerca de 20 millones de /año (0,097-0,12\% del gasto global en Asistencia Especializada).

\section{Bibliografía}

1. Pineda L, Hathwar VS, Grant BJ. Clinical suspicion of fatal pulmonary embolism. Chest 2001; 120: 791-5.

2. Dalen JE. Pulmonary embolism: what have we learned since Virchow? Natural history, pathophisiology and diagnosis. Chest 2002; 122: 144056.

3. Saro G, Campos JF, Hernández JM, Anta M, Olmos JM, GonzálezMacías et al. Diagnostic approach to patients with suspected pulmonary embolism: a report from the real world. Postgrad Med J 1999; 75: 2859.

4. Douketis JD, Kearon C, Bates S, Duku EK, Ginsberg JC. Risk of fatal pulmonary embolism in patients with treated venous thromboembolism. JAMA 1998; 279: 458-62.

5. Suárez C. Tratamiento ambulatorio de la enfermedad tromboembólica venosa. Med Clin (Barc) 2002; 3 (Supl. 2): 43-5.

6. Hanson PO, Welin L, Tibblin G, Eriksson H. Deep vein thrombosis and pulmonary embolism in the general population. Arch Intern Med 1997; 157: $1665-70$

7. Silverstein MD, Heit JA, Mohr DN, Petterson TM, O’Fallon WN, Melton LJIII. Trends in the incidence of deep vein thromboses and pulmonary embolism: a 25-year population-based study. Arch Intern Med 1998; 158: 585-93.

8. Heit J, Melton LJ, Lohse CM, Petterson TM, Silverstein MD, Mohr DN et al. Incidence of venous thromboembolism in hospitalized patients vs. community residents. Mayo Clin Proc 2001; 76: 1102-10.

9. Goldhaber SZ. Pulmonary embolism. N Engl J Med 1998; 339: 93-104.

10. Rocha E, Panizo C, Lecumberri R, Aranguren A, Cuesta B, Hermida J. Tratamiento de la tromboembolia pulmonar. Med Clin (Barc) 2002; 3 (Supl. 2):46-53.

11. Warner GT, Perry CM, Ferguson JT. Enoxaparin in the prevention of tromboembolic disease in medical patients. Am J Cardiovasc Drugs 2001; 1: 477-82.

12. Monreal M. Epidemiología, patogenia e historia natural del tromboembolismo venoso. En: Rocha Hernando E, Martínez Brotons F, Monreal Bosch M. Manejo práctico del paciente con tromboembolismo venoso. Madrid: Acción Médica; 2002; 1-10.

13. Gabriel Botella F. Reflexiones sobre la enfermedad tromboembólica venosa. An Med Interna (Madrid) 2003; 20: 447-50.

14. Arnalich Hernández F, Fernández Pavón A. Sospecha y diagnóstico del trombembolismo pulmonar en la actualidad. Rev Clin Esp 2002; 202:127-9

15. Guidelines on diagnosis and management of acute pulmonary embolism. Task-force on pulmonary embolism. European Society of Cardiology. Eur Heart J 2000; 21: 1301-6.
16. Calvo Romero JM, Pérez Miranda M, Bureo Dacal P. Tromboembolismo pulmonar en el anciano. An Med Interna Madrid 2003; 20: 21-4.

17. Echegaray Agara M, Alonso Martínez JL, Urbieta Echezarreta MA, Abinzano Guillén ML, González Arencibia C, Solano Remírez M. Tromboembolismo pulmonar: evolución a largo plazo y epidemiología clínica. An Med Interna (Madrid) 2003; 20: 451-6.

18. Ministerio de Sanidad y Consumo. CMBD/GDR-SNS. (Disponible en www.msc.es/explotación).

19. Sistema Nacional de Salud. Año 1999. Explotación de bases del CMBD. Estadísticos de referencia estatal de los sistemas de agrupación de registros de pacientes (G.D.R.). Ministerio de Sanidad y Consumo, Subdirección general de Análisis Económico y Estadística; Madrid 2002.

20. Conxunto Mínimo Básico de Datos. Hospitais do Servicio Galego de Saúde. Varios volúmenes: Bienios 1996-2000. Subdirección Xeral de Información Sanitaria. SERGAS.

21. Ministerio de Sanidad y Consumo. Sistema Nacional de Salud en cifras, 2002. (Disponible en http://www.msc.es/planificacion.sanitaria/estadisticas.sanitarias/cifras)

22. López-Beret P, Pinto JM, Romero A, Orgaz A, Fontcuberta J, Oblas M. Systematic study of occult pulmonary thromboembolism in patients with deep venous thhombosis. J Vasc Surg 2001; 33: 515-21.

23. Meignan M, Rosso J, Gauthier H, Brunengo F, Claudel S, Sagnard L et al. Systematic lung scans reveal a high frequency of silent pulmonary embolism in patients with proximal deep venous thrombosis. Arch Intern Med 2000; 160: 159-64.

24. Eaglen C, Ker JP. Deep vein thrombosis/pulmonary embolism (Disponible en http:/www.dresources.com; acceso 16/11/ 2002).

25. Bergqvist, David. Enoxaparin: a pharmaeconomic review of its use in the prevention and treatment of venous thromboembolism and in acute coronary syndrome. Pharmaeconomics 2002; 20: 225-43.

26. Geerts WH, Heit JA, Clagett GP, Pineo GF, Colwell CW, Anderson, Jr FA, Wheeler HB. Prevention of venous thomboembolism. Chest 2001; 119: $132 \mathrm{~S}-75 \mathrm{~S}$.)

27. Otero R, Uresandi F, Cayuela A, Blanquer J, Cabezudo MA, De Gregorio MA et al. Use of venous thromboembolism prophylaxis for surgical patients: a multicentre analysis of practice in Spain. Eur J Surg 2001; 167: 163-7.

28. Stein PD, Huang HL, Afzal A, Noor HA. Incidence of acute pulmonary embolism in a General Hospital. Relation to age, sex and race. Chest. 1999; 116: 909-13.

29. Stein PD, Patel KC, Kalra NK, Petrina M, Savarapu P, Furlong Jr. JW, et al. Estimated incidence of acute pulmonary embolism in a community/teaching general hospital Chest 2002; 121: 802-5. 
30. Meaney JF, Weg JG, Chenevert TL, Stafford-Johnson D, Hamilton BH, Prince MR. Diagnosis of pulmonary embolism with magnetic resonance angiography. N Engl J Med 1997; 336: 1422-7.

31. American Thoracic Society. The diagnostic approach to acute venous thromboembolism. Am J Respir Crit Care Med 1999; 160: 1043-66.

32. Baile EM, King GG, Müller NL, D'Yachkova Y, Coche EE, Paré PD, Mayo JR. Spiral computed tomography is comparable to angiography for the diagnosis of pulmonary embolism. Am J Respir Crit Care Med 2000; 161: 1010-15.

33. Lorut C, Ghossains M, Horellou ME, Achkar A, Fretault J, Laaban JP. A Noninvasive Diagnostic Strategy Including Spiral Computed Tomography in Patients with Suspected Pulmonary Embolism. Am J Respir Crit Care Med 2000; 162 (4 Pt 1):1413-8.

34. Hyers TM, Agnelli G, Hull RD, Morris TA, Samama M, Tapson V, Weg JC. Antithrombotic Therapy for Venous Thromboembolic Disease. Chest 2001; 119: 176S-193S.
35. Girard P, Musset D, Parent F, Maitre S, Philippotteau C, Simmonneau G. High prevalence of detectable deep venous thrombosis in patients with acute pulmonary embolism. Chest 1999; 116: 903-8.

36. Goldhaber SZ, Grodstein F, Stampler MJ, Manson JE, Colditz GA, Speizer FE et al. A prospective study of risk factors for pulmonary embolism in women. JAMA 1997; 277: 642-5.

37. Hansson PO, Eriksson H, Welin L, Svärdsudd K, Wilhemsen L. Smoking and abdominal obesity. Risk factors for venous thromboembolism among middle-aged men: "The study of men born in 1913". Arch Intern Med 1999; 159: 1886-90.

38. Suárez Fernández C, González-Fajardo JA, Monreal Bosch M y Grupo de Trabajo de Enfermedad Tromboembólica en España. Registro informatizado de pacientes con enfermedad tromboembólica venosa en España: un esfuerzo al servicio del médico y del paciente. Med Clin (Barc) 2002; 3 (Supl. 2): 1-2. 